Vitrinite reflectance data for the Greater Green River Basin, southwestern Wyoming, northwestern Colorado, and northeastern Utah

By Mark J. Pawlewicz ${ }^{1}$ and Thomas M. Finn ${ }^{1}$

Open-File Report 02-339

2002

This report is preliminary and has not been reviewed for conformity with U.S. Geological Survey editorial standards or with the North American Stratigraphic Code. Any use of trade, firm, or product names is for descriptive purposes only and does not imply endorsement by the U.S. Government.

U.S. DEPARTMENT OF THE INTERIOR U.S. GEOLOGICAL SURVEY

${ }^{1}$ Denver, Colorado 


\title{
Vitrinite reflectance (Ro) data for the Greater Green River Basin, southwestern Wyoming, northwestern Colorado, and northeastern Utah
}

by

\author{
Mark J. Pawlewicz and Thomas M. Finn
}

The Greater Green River Basin is a large Laramide (Late Cretaceous through

Eocene) structural and sedimentary basin that encompasses about 25,000 square miles in southwestern Wyoming, northwestern Colorado, and northeastern Utah (fig. 1).

Important conventional oil and gas resources have been discovered and produced from reservoirs ranging in age from Cambrian through Tertiary (Law, 1996). In addition, an extensive overpressured basin - centered gas accumulation has also been identified in Cretaceous and Tertiary reservoirs by numerous researchers including Law (1984a, 1996), Law and others (1980, 1989), McPeek (1981), and Spencer (1987). The purpose of this report is to present new vitrinite reflectance data to be used in support of the U.S Geological Survey assessment of undiscovered oil and gas resources of the Greater Green River Basin.

One hundred eighty-six samples were collected from Cretaceous and Tertiary coalbearing strata (figs. 1 and 2) in an effort to better understand and characterize the thermal maturation and burial history of potential source rocks. Two samples were from core, one from outcrop, and the remainder from well cuttings. These data were collected to supplement previously published data by Law (1984b), Pawlewicz and others (1986), Merewether and others (1987), and Garcia-Gonzalez and Surdam (1995) and are presented in table 1. 
The coal samples were analyzed by vitrinite reflectance to determine levels of thermal maturation. Preparation of the coal for reflectance analysis required (1) crushing the larger coal pieces into 0.25 to $1 \mathrm{~mm}$ pieces, (2) casting the pieces with epoxy in precut and drilled plugs, and (3) curing the samples overnight. Subsequently, a four-step grinding process was implemented - two with progressively finer sandpaper (60 and 600 grit), and two polishing steps ( 0.3 and 0.05 micron). Vitrinite reflectance measurements were determined at $500 \mathrm{X}$ magnification using plane-polarized incident white light and a $546 \mathrm{~nm}$ monochromatic filter in immersion oil. For samples with sufficient quality vitrinite, at least 25 measurements were recorded. For samples of poorer quality, either due to a poor polish or to the presence of mineral or other inorganic material, fewer measurements were recorded. 


\section{References}

Garcia-Gonzalez, M., and Surdam, R.C., 1995, Hydrocarbon generation potential and expulsion efficency in shales and coals: example from the Washakie Basin, Wyoming; in Jones, R.W., ed., Resources of southwestern Wyoming, Wyoming Geological Association Guidebook, p. 225-245.

Law, B.E., 1984a, Geological characterization of low-permeability Upper Cretaceous and Lower Tertiary rocks in the Pinedale Anticline area, Sublette County, Wyoming: U.S. Geological Survey Open File Report 84-753, 107 p.

, 1984b, Relationships of source-rock, thermal maturity, and overpressuring to gas generation and occurrence in low-permeability Upper Cretaceous and Lower Tertiary rocks, Greater Green River Basin, Wyoming, Colorado, and Utah; in $\square$ Woodward, J., Meissner, F.F., and Clayton, J.L., eds., Hydrocarbon source rocks of the greater Rocky Mountain region: Rocky Mountain Association of Geologists Guidebook, p. 469-490.

, 1996, Southwestern Wyoming Province; in Gautier, D.L., Dolton, G.L.,

Takahashi, K.I., and Varnes, K.L., eds., 1995 National assessment of United States oil and gas resources-results, methodology, and supporting data: U.S. Geological Survey Digital Data Series DDS-30, release 2. 
Law, B.E., Spencer, C.W., and Bostick, N.H., 1980, Evaluation of organic matter, subsurface temperature and pressure with regard to gas generation in lowpermeability Upper Cretaceous and Lower Tertiary sandstones in the Pacific Creek area, Sublette and Sweetwater Counties, Wyoming: The Mountain Geologist, v. 17, no. 2, p. 23-35.

Law, B.E., Spencer, C.W., Charpentier, R.R., Crovelli, R.A., Mast, R.F., Dolton, G.L., and Wandrey, C.J., 1989, Estimates of gas resources in overpressured lowpermeability Cretaceous and Tertiary sandstone reservoirs, Greater Green River Basin, Wyoming, Colorado, and Utah; in Eisert, J.L., ed., Gas Resources of Wyoming: Wyoming Geological Association Fortieth Field Conference Guidebook, p. 39-61.

McPeek, L.A., 1981, Eastern Green River Basin: a developing giant gas supply from deep, overpressured Upper Cretaceous sandstones: American Association of Petroleum Geologists Bulletin, v. 65, no. 6, p. 1078-1098.

Merewether, E.A., Krystinik, K.B., and Pawlewicz, M.J., 1987, Thermal maturity of hydrocarbon-bearing formations in southwestern Wyoming and northwestern Colorado: U.S. Geological Survey Miscellaneous Investigations Series Map I$1831,1: 500,000,1$ sheet. 
Pawlewicz, M.J., Licus, M.R., Law, B.E., Dickinson, W.W., and Barclay, C.S.V., 1986, Thermal maturity map showing subsurface elevation of 0.8 percent vitrinite reflectance in the Greater Green River Basin of Wyoming, Colorado, and Utah: U.S. Geological Survey Miscellaneous Field Studies Map MF-1890, 1:500,000, 1 sheet.

Spencer, C.W., 1987, Hydrocarbon generation as a mechanism for overpressuring in the Rocky Mountain region: American Association of Petroleum Geologists Bulletin, v. 71 , no. 4 , p. $368-388$. 
Figure 1. Map showing the general outline of the Greater Green River Basin, and sample localities. Numbers refer to column 1 in Table 1.

Figure 2. Generalized stratigraphic chart of Cretaceous and lower Tertiary rocks in the Greater Green River Basin (modified from Law, 1996). 
Table 1. Vitrinite reflectance (Ro) data and sample locations for the Greater Green River Basin; southwestern Wyoming, northwestern Colorado, and northeastern Utah. Map no. (column 1) refers to numbers plotted on figure 1. [Tnsp = Township; Rng $=$ Range; Sec $=$ Section; Lith $=$ Lithology carb sh $=$ carconaceous shale; Std . dev. $=$ Standard deviation; Fm/Grp $=$ Formation/Group. Queried (?) where uncertain; $\mathrm{E}=$ East $; \mathrm{W}=$ West $\mathrm{N}=$ North; $\mathrm{nd}=$ no determination; $\mathrm{n}=$ number of readings per sample. Depth is in feet]. 


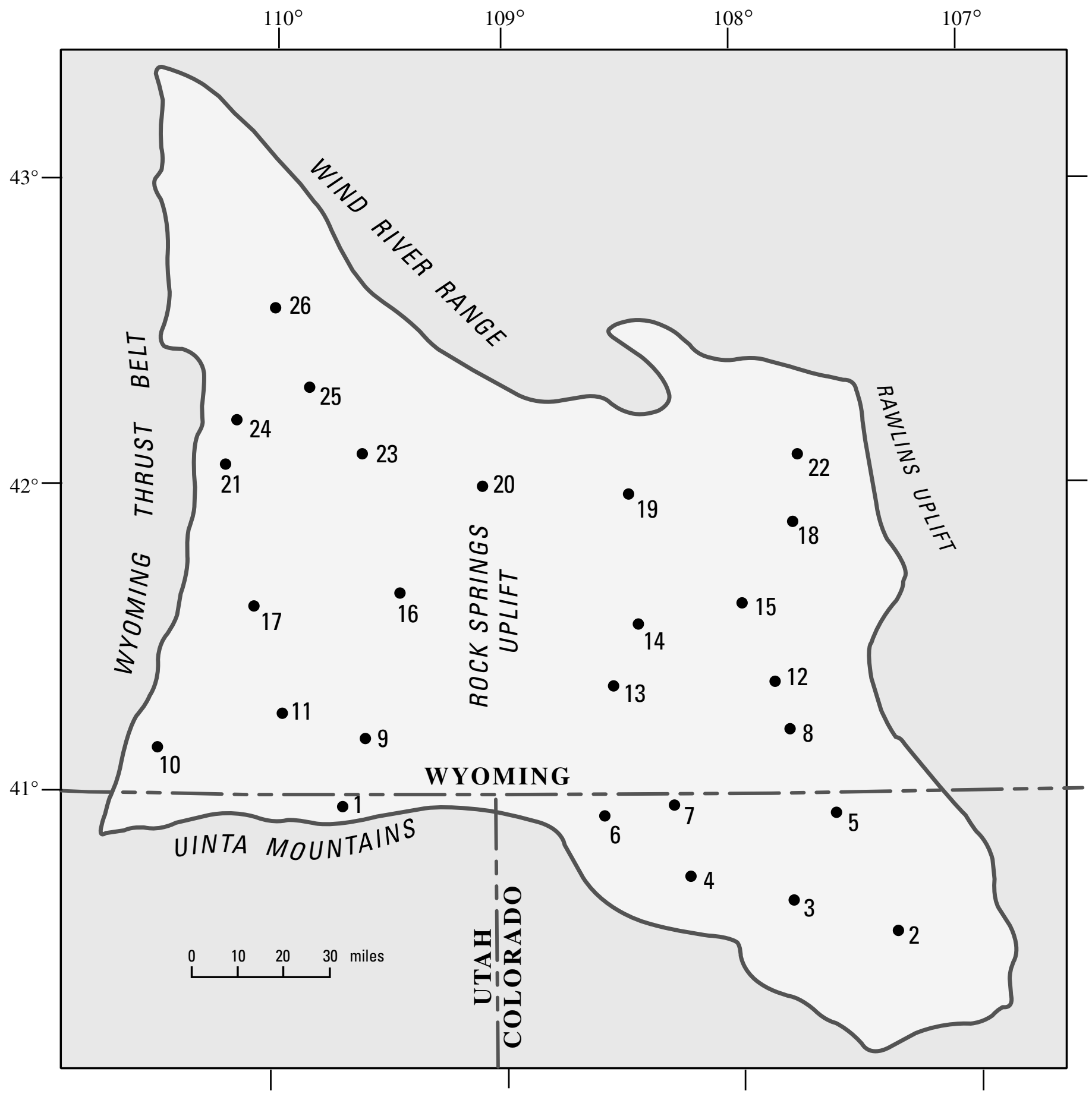




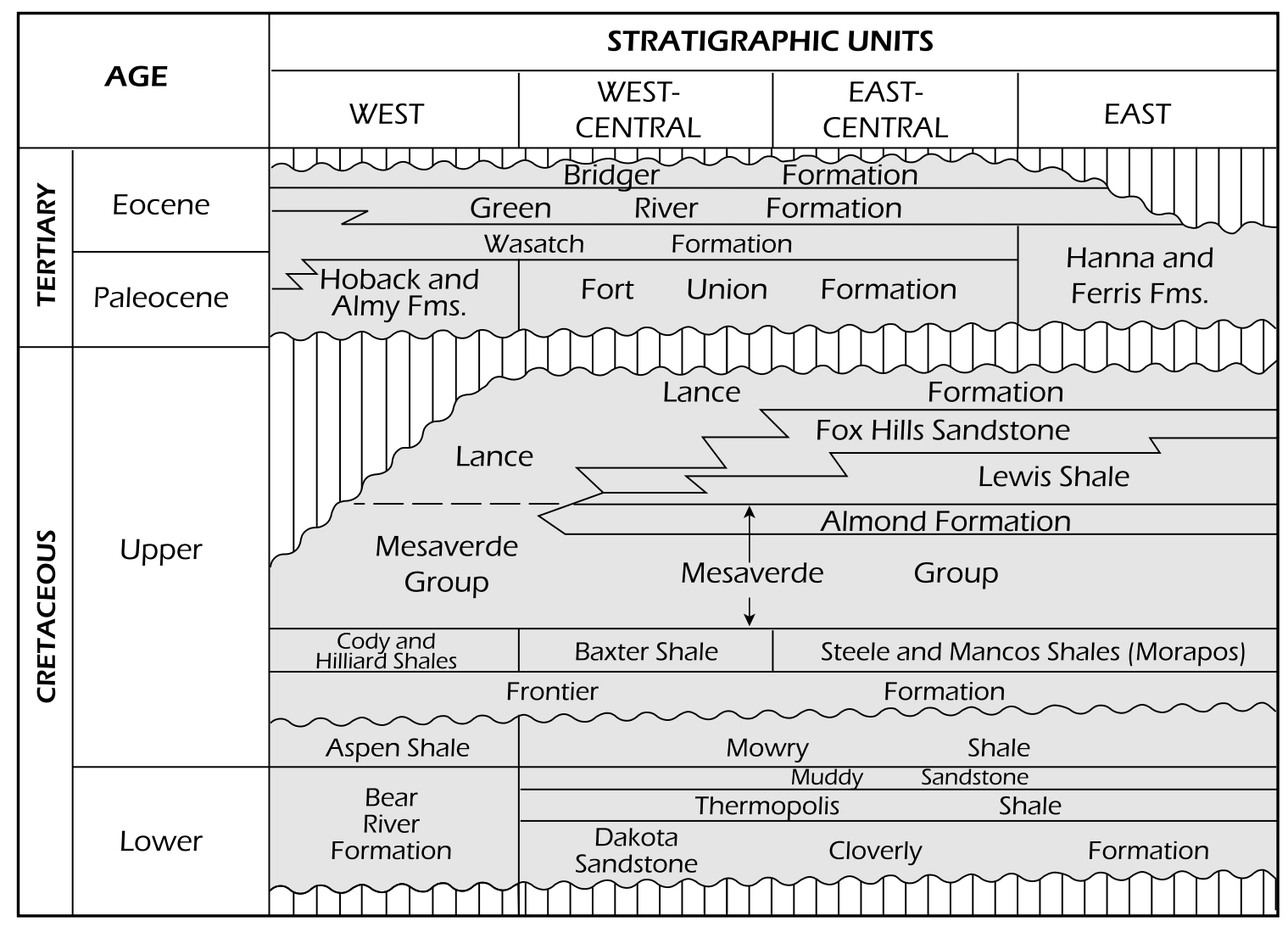




\begin{tabular}{|c|c|c|c|c|c|c|c|c|c|c|c|c|c|}
\hline Map no. & Operator & Well name & State & Tnsp & Rng & Sec & Sample type & Lith & Depth & \%Ro & $\mathbf{n}$ & Std. dev. & Fm/Grp \\
\hline 1 & Outcrop & 2001-UT-1 & UT & $3 \mathrm{~N}$ & $20 \mathrm{E}$ & 31 & outcrop & coal & surface & 0.54 & 35 & 0.02 & Dakota \\
\hline 2 & Texas Pacific & 1 Bear & $\mathrm{CO}$ & $7 \mathrm{~N}$ & $89 \mathrm{~W}$ & 26 & cuttings & coal & $370-400$ & 0.42 & 7 & 0.01 & Lance \\
\hline 2 & Texas Pacific & 1 Bear & $\mathrm{CO}$ & $7 \mathrm{~N}$ & $89 \mathrm{~W}$ & 26 & cuttings & coal & $790-820$ & 0.41 & 21 & 0.03 & Lance \\
\hline 2 & Texas Pacific & 1 Bear & $\mathrm{CO}$ & $7 \mathrm{~N}$ & $89 \mathrm{~W}$ & 26 & cuttings & coal & $3800-3810$ & 0.68 & 25 & 0.03 & Mesaverde \\
\hline 2 & Texas Pacific & 1 Bear & $\mathrm{CO}$ & $7 \mathrm{~N}$ & $89 \mathrm{~W}$ & 26 & cuttings & coal & $4300-4310$ & 0.68 & 15 & 0.04 & Mesaverde \\
\hline 2 & Texas Pacific & 1 Bear & $\mathrm{CO}$ & $7 \mathrm{~N}$ & $89 \mathrm{~W}$ & 26 & cuttings & coal & $4800-4810$ & 0.7 & 27 & 0.04 & Mesaverde \\
\hline 2 & Texas Pacific & 1 Bear & $\mathrm{CO}$ & $7 \mathrm{~N}$ & $89 \mathrm{~W}$ & 26 & cuttings & coal & $5040-5050$ & 0.74 & 29 & 0.04 & Mesaverde \\
\hline 2 & Texas Pacific & 1 Bear & $\mathrm{CO}$ & $7 \mathrm{~N}$ & $89 \mathrm{~W}$ & 26 & cuttings & coal & $5350-5360$ & 0.71 & 27 & 0.04 & Mesaverde \\
\hline 2 & Texas Pacific & 1 Bear & $\mathrm{CO}$ & $7 \mathrm{~N}$ & $89 \mathrm{~W}$ & 26 & cuttings & coal & $6200-6210$ & 0.71 & 31 & 0.04 & Mesaverde \\
\hline 2 & Texas Pacific & 1 Bear & $\mathrm{CO}$ & $7 \mathrm{~N}$ & $89 \mathrm{~W}$ & 26 & cuttings & coal & $6510-6520$ & 0.76 & 27 & 0.04 & Mesaverde \\
\hline 2 & Texas Pacific & 1 Bear & $\mathrm{CO}$ & $7 \mathrm{~N}$ & $89 \mathrm{~W}$ & 26 & cuttings & coal & $6800-6810$ & 0.67 & 23 & 0.05 & Mesaverde \\
\hline 2 & Texas Pacific & 1 Bear & $\mathrm{CO}$ & $7 \mathrm{~N}$ & $89 \mathrm{~W}$ & 26 & cuttings & coal & $11050-11060$ & 0.71 & 12 & 0.04 & Frontier \\
\hline 3 & Humble & 1 Lay Creek & $\mathrm{CO}$ & $8 \mathrm{~N}$ & $93 \mathrm{~W}$ & 13 & cuttings & coal & $960-970$ & 0.52 & 25 & 0.06 & Fort Union \\
\hline 3 & Humble & 1 Lay Creek & $\mathrm{CO}$ & $8 \mathrm{~N}$ & $93 \mathrm{~W}$ & 13 & cuttings & coal & $1190-1220$ & 0.56 & 25 & 0.03 & Fort Union \\
\hline 3 & Humble & 1 Lay Creek & $\mathrm{CO}$ & $8 \mathrm{~N}$ & $93 \mathrm{~W}$ & 13 & cuttings & coal & $1500-1540$ & 0.59 & 27 & 0.03 & Fort Union \\
\hline 3 & Humble & 1 Lay Creek & $\mathrm{CO}$ & $8 \mathrm{~N}$ & $93 \mathrm{~W}$ & 13 & cuttings & coal & $2040-2050$ & 0.55 & 25 & 0.03 & Lance \\
\hline 3 & Humble & 1 Lay Creek & $\mathrm{CO}$ & $8 \mathrm{~N}$ & $93 \mathrm{~W}$ & 13 & cuttings & coal & $4280-4290$ & 0.75 & 27 & 0.03 & Mesaverde \\
\hline 3 & Humble & 1 Lay Creek & $\mathrm{CO}$ & $8 \mathrm{~N}$ & $93 \mathrm{~W}$ & 13 & cuttings & coal & $4760-4770$ & 0.75 & 29 & 0.04 & Mesaverde \\
\hline 3 & Humble & 1 Lay Creek & $\mathrm{CO}$ & $8 \mathrm{~N}$ & $93 \mathrm{~W}$ & 13 & cuttings & coal & $5240-5260$ & 0.78 & 25 & 0.06 & Mesaverde \\
\hline 3 & Humble & 1 Lay Creek & $\mathrm{CO}$ & $8 \mathrm{~N}$ & $93 \mathrm{~W}$ & 13 & cuttings & coal & $6620-6660$ & 0.88 & 21 & 0.05 & Mesaverde \\
\hline 4 & C \& K Petroleum & 1-19 Gulf Federal & $\mathrm{CO}$ & $9 \mathrm{~N}$ & $96 \mathrm{~W}$ & 19 & cuttings & coal & $7360-7590$ & 0.56 & 25 & 0.07 & Fort Union \\
\hline 4 & C \& K Petroleum & 1-19 Gulf Federal & $\mathrm{CO}$ & $9 \mathrm{~N}$ & $96 \mathrm{~W}$ & 19 & cuttings & coal & $83108-570$ & 0.75 & 29 & 0.04 & Fort Union \\
\hline 4 & C \& K Petroleum & 1-19 Gulf Federal & $\mathrm{CO}$ & $9 \mathrm{~N}$ & $96 \mathrm{~W}$ & 19 & cuttings & coal & $8700-8820$ & 0.8 & 21 & 0.07 & Fort Union \\
\hline 4 & C \& K Petroleum & 1-19 Gulf Federal & $\mathrm{CO}$ & $9 \mathrm{~N}$ & $96 \mathrm{~W}$ & 19 & cuttings & coal & $9180-91400$ & 0.71 & 25 & 0.04 & Lance \\
\hline 4 & C \& K Petroleum & 1-19 Gulf Federal & $\mathrm{CO}$ & $9 \mathrm{~N}$ & $96 \mathrm{~W}$ & 19 & cuttings & coal & $11000-12210$ & 0.93 & 19 & 0.04 & Mesaverde \\
\hline 5 & Superior Oil & 1 State & $\mathrm{CO}$ & $11 \mathrm{~N}$ & $91 \mathrm{~W}$ & 3 & cuttings & coal & $2190-2210$ & 0.53 & 25 & 0.03 & Fort Union \\
\hline 5 & Superior Oil & 1 State & $\mathrm{CO}$ & $11 \mathrm{~N}$ & $91 \mathrm{~W}$ & 3 & cuttings & coal & $2900-3010$ & 0.55 & 27 & 0.06 & Lance \\
\hline 5 & Superior Oil & 1 State & $\mathrm{CO}$ & $11 \mathrm{~N}$ & $91 \mathrm{~W}$ & 3 & cuttings & coal & $6400-6430$ & 0.73 & 31 & 0.05 & Mesaverde \\
\hline 5 & Superior Oil & 1 State & $\mathrm{CO}$ & $11 \mathrm{~N}$ & $91 \mathrm{~W}$ & 3 & cuttings & coal & $6680-6730$ & 0.71 & 25 & 0.04 & Mesaverde \\
\hline 5 & Superior Oil & 1 State & $\mathrm{CO}$ & $11 \mathrm{~N}$ & $91 \mathrm{~W}$ & 3 & cuttings & coal & $8100-8130$ & 0.76 & 25 & 0.05 & Mesaverde \\
\hline 6 & Champlin & 1 USA 41-12 & $\mathrm{CO}$ & $11 \mathrm{~N}$ & $100 \mathrm{~W}$ & 12 & cuttings & coal & $3650-3660$ & 0.63 & 31 & 0.05 & Fort Union \\
\hline 6 & Champlin & 1 USA 41-12 & $\mathrm{CO}$ & $11 \mathrm{~N}$ & $100 \mathrm{~W}$ & 12 & cuttings & coal & $4400-4410$ & 0.56 & 29 & 0.05 & Fort Union \\
\hline 6 & Champlin & 1 USA 41-12 & $\mathrm{CO}$ & $11 \mathrm{~N}$ & $100 \mathrm{~W}$ & 12 & cuttings & coal & $5100-5140$ & 0.56 & 35 & 0.03 & Fort Union \\
\hline 6 & Champlin & 1 USA 41-12 & $\mathrm{CO}$ & $11 \mathrm{~N}$ & $100 \mathrm{~W}$ & 12 & cuttings & coal & $5950-5970$ & 0.71 & 19 & 0.06 & Lance \\
\hline 6 & Champlin & 1 USA 41-12 & $\mathrm{CO}$ & $11 \mathrm{~N}$ & $100 \mathrm{~W}$ & 12 & cuttings & coal & $7230-7250$ & 0.75 & 21 & 0.08 & Mesaverde \\
\hline
\end{tabular}




\begin{tabular}{|c|c|c|c|c|c|c|c|c|c|c|c|c|c|}
\hline 6 & Champlin & 1 USA 41-12 & $\mathrm{CO}$ & $11 \mathrm{~N}$ & $100 \mathrm{~W}$ & 12 & cuttings & coal & $7900-7940$ & 0.9 & 35 & 0.04 & Mesaverde \\
\hline 6 & Champlin & 1 USA 41-12 & $\mathrm{CO}$ & $11 \mathrm{~N}$ & $100 \mathrm{~W}$ & 12 & cuttings & coal & $8280-8300$ & 0.9 & 31 & 0.06 & Mesaverde \\
\hline 6 & Champlin & 1 USA 41-12 & $\mathrm{CO}$ & $11 \mathrm{~N}$ & $100 \mathrm{~W}$ & 12 & cuttings & coal & $8570-8610$ & 0.84 & 35 & 0.05 & Mesaverde \\
\hline 6 & Champlin & 1 USA 41-12 & $\mathrm{CO}$ & $11 \mathrm{~N}$ & $100 \mathrm{~W}$ & 12 & cuttings & coal & $9200-9240$ & 0.83 & 5 & 0.09 & Mesaverde \\
\hline 6 & Champlin & 1 USA 41-12 & $\mathrm{CO}$ & $11 \mathrm{~N}$ & $100 \mathrm{~W}$ & 12 & cuttings & coal & $15400-15460$ & 2.35 & 14 & 0.15 & Dakota \\
\hline 7 & Mountain Fuel & 11 Carl Allen & $\mathrm{CO}$ & $12 \mathrm{~N}$ & $97 \mathrm{~W}$ & 33 & cuttings & coal & $5460-5500$ & 0.58 & 25 & 0.04 & Fort Union \\
\hline 7 & Mountain Fuel & 11 Carl Allen & $\mathrm{CO}$ & $12 \mathrm{~N}$ & $97 \mathrm{~W}$ & 33 & cuttings & coal & $6100-6180$ & 0.55 & 25 & 0.03 & Fort Union \\
\hline 7 & Mountain Fuel & 11 Carl Allen & $\mathrm{CO}$ & $12 \mathrm{~N}$ & $97 \mathrm{~W}$ & 33 & cuttings & coal & $6690-6750$ & 0.65 & 25 & 0.03 & Fort Union \\
\hline 7 & Mountain Fuel & 11 Carl Allen & $\mathrm{CO}$ & $12 \mathrm{~N}$ & $97 \mathrm{~W}$ & 33 & cuttings & coal & $8610-8690$ & 0.69 & 23 & 0.1 & Lance \\
\hline 7 & Mountain Fuel & 11 Carl Allen & $\mathrm{CO}$ & $12 \mathrm{~N}$ & $97 \mathrm{~W}$ & 33 & cuttings & coal & $11020-11040$ & 1.26 & 26 & 0.04 & Mesaverde \\
\hline 7 & Mountain Fuel & 11 Carl Allen & $\mathrm{CO}$ & $12 \mathrm{~N}$ & $97 \mathrm{~W}$ & 33 & cuttings & coal & $11660-11700$ & 1.25 & 27 & 0.04 & Mesaverde \\
\hline 7 & Mountain Fuel & 11 Carl Allen & $\mathrm{CO}$ & $12 \mathrm{~N}$ & $97 \mathrm{~W}$ & 33 & cuttings & coal & $12610-12650$ & 1.26 & 25 & 0.07 & Mesaverde \\
\hline 8 & CIG Exploration & 6-14-92 Blue Gap II & WY & $14 \mathrm{~N}$ & $92 \mathrm{~W}$ & 6 & cuttings & coal & $3900-3990$ & 0.51 & 13 & 0.04 & Fort Union \\
\hline 8 & CIG Exploration & 6-14-92 Blue Gap II & WY & $14 \mathrm{~N}$ & $92 \mathrm{~W}$ & 6 & cuttings & coal & $4770-4830$ & 0.66 & 31 & 0.03 & Fort Union \\
\hline 8 & CIG Exploration & 6-14-92 Blue Gap II & WY & $14 \mathrm{~N}$ & $92 \mathrm{~W}$ & 6 & cuttings & coal & $5370-5430$ & 0.66 & 31 & 0.04 & Lance \\
\hline 8 & CIG Exploration & 6-14-92 Blue Gap II & WY & $14 \mathrm{~N}$ & $92 \mathrm{~W}$ & 6 & cuttings & coal & $6430-6460$ & 0.76 & 31 & 0.06 & Lance \\
\hline 8 & CIG Exploration & 6-14-92 Blue Gap II & WY & $14 \mathrm{~N}$ & $92 \mathrm{~W}$ & 6 & cuttings & coal & $9100-9150$ & 0.97 & 31 & 0.04 & Mesaverde \\
\hline 8 & CIG Exploration & 6-14-92 Blue Gap II & WY & $14 \mathrm{~N}$ & $92 \mathrm{~W}$ & 6 & cuttings & coal & $9900-9960$ & 1.06 & 31 & 0.07 & Mesaverde \\
\hline 8 & CIG Exploration & 6-14-92 Blue Gap II & WY & $14 \mathrm{~N}$ & $92 \mathrm{~W}$ & 6 & cuttings & coal & $11340-11390$ & 1.33 & 35 & 0.14 & Mesaverde \\
\hline 9 & Tom Brown Inc. & 1 Currant Creek Fed. & WY & $14 \mathrm{~N}$ & $108 \mathrm{~W}$ & 20 & cuttings & carb sh & $2950-3040$ & nd & nd & nd & nd \\
\hline 9 & Tom Brown Inc. & 1 Currant Creek Fed. & WY & $14 \mathrm{~N}$ & $108 \mathrm{~W}$ & 20 & cuttings & coal & $4490-4600$ & 0.7 & 10 & 0.11 & Wasatch(?) \\
\hline 9 & Tom Brown Inc. & 1 Currant Creek Fed. & WY & $14 \mathrm{~N}$ & $108 \mathrm{~W}$ & 20 & cuttings & coal & $6180-6240$ & 0.59 & 15 & 0.08 & Fort Union \\
\hline 9 & Tom Brown Inc. & 1 Currant Creek Fed. & WY & $14 \mathrm{~N}$ & $108 \mathrm{~W}$ & 20 & cuttings & coal & $7090-7140$ & 0.59 & 21 & 0.05 & Fort Union \\
\hline 9 & Tom Brown Inc. & 1 Currant Creek Fed. & WY & $14 \mathrm{~N}$ & $108 \mathrm{~W}$ & 20 & cuttings & coal & $9050-9180$ & 0.74 & 27 & 0.05 & Mesaverde \\
\hline 9 & Tom Brown Inc. & 1 Currant Creek Fed. & WY & $14 \mathrm{~N}$ & $108 \mathrm{~W}$ & 20 & cuttings & coal & $10400-10470$ & 0.95 & 25 & 0.05 & Mesaverde \\
\hline 9 & Tom Brown Inc. & 1 Currant Creek Fed. & WY & $14 \mathrm{~N}$ & $108 \mathrm{~W}$ & 20 & cuttings & coal & $16800-16890$ & 1.93 & 21 & 0.12 & Frontier \\
\hline 9 & Tom Brown Inc. & 1 Currant Creek Fed. & WY & $14 \mathrm{~N}$ & $108 \mathrm{~W}$ & 20 & cuttings & carb sh & $17280-17300$ & 2.89 & 5 & 0.37 & Dakota \\
\hline 10 & Sun Oil Company & 1 Blacks Fork Unit & WY & $14 \mathrm{~N}$ & $116 \mathrm{~W}$ & 34 & cuttings & coal & $7130-7190$ & 0.54 & 25 & 0.04 & Fort Union \\
\hline 10 & Sun Oil Company & 1 Blacks Fork Unit & WY & $14 \mathrm{~N}$ & $116 \mathrm{~W}$ & 34 & cuttings & coal & $7480-7530$ & 0.55 & 25 & 0.04 & Fort Union \\
\hline 10 & Sun Oil Company & 1 Blacks Fork Unit & WY & $14 \mathrm{~N}$ & $116 \mathrm{~W}$ & 34 & cuttings & coal & $7870-7930$ & 0.57 & 31 & 0.06 & Fort Union \\
\hline 10 & Sun Oil Company & 1 Blacks Fork Unit & WY & $14 \mathrm{~N}$ & $116 \mathrm{~W}$ & 34 & cuttings & coal & $8140-8210$ & 0.56 & 31 & 0.05 & Fort Union \\
\hline 10 & Sun Oil Company & 1 Blacks Fork Unit & WY & $14 \mathrm{~N}$ & $116 \mathrm{~W}$ & 34 & cuttings & coal & $10470-10550$ & 0.61 & 31 & 0.03 & Mesaverde \\
\hline 10 & Sun Oil Company & 1 Blacks Fork Unit & WY & $14 \mathrm{~N}$ & $116 \mathrm{~W}$ & 34 & cuttings & coal & $14790-14800$ & 1.01 & 35 & 0.03 & Frontier \\
\hline 11 & Texaco & 1 Leo Unit & WY & $15 \mathrm{~N}$ & $111 \mathrm{~W}$ & 20 & cuttings & coal & $6400-6430$ & 0.78 & 10 & 0.04 & Fort Union \\
\hline 11 & Texaco & 1 Leo Unit & WY & $15 \mathrm{~N}$ & $111 \mathrm{~W}$ & 20 & cuttings & coal & $6970-7030$ & 0.57 & 25 & 0.06 & Fort Union \\
\hline 11 & Texaco & 1 Leo Unit & WY & $15 \mathrm{~N}$ & $111 \mathrm{~W}$ & 20 & cuttings & coal & $7660-7750$ & 0.6 & 27 & 0.05 & Fort Union \\
\hline 11 & Texaco & 1 Leo Unit & WY & $15 \mathrm{~N}$ & $111 \mathrm{~W}$ & 20 & cuttings & coal & $8220-8240$ & 0.68 & 31 & 0.06 & Fort Union \\
\hline
\end{tabular}




\begin{tabular}{|c|c|c|c|c|c|c|c|c|c|c|c|c|c|}
\hline 11 & Texaco & 1 Leo Unit & $\overline{W Y}$ & $15 \mathrm{~N}$ & $111 \mathrm{~W}$ & 20 & cuttings & coal & $8690-8720$ & 0.72 & 31 & 0.05 & Fort Union \\
\hline 11 & Texaco & 1 Leo Unit & WY & $15 \mathrm{~N}$ & $111 \mathrm{~W}$ & 20 & cuttings & coal & $9610-9680$ & 0.77 & 19 & 0.03 & Mesaverde \\
\hline 11 & Texaco & 1 Leo Unit & WY & $15 \mathrm{~N}$ & $111 \mathrm{~W}$ & 20 & cuttings & coal & $10130-10140$ & 0.72 & 33 & 0.02 & Mesaverde \\
\hline 12 & Ambassador oil & 1 Federal-J-Wyoming & WY & $16 \mathrm{~N}$ & $93 \mathrm{~W}$ & 14 & cuttings & coal & $2320-2380$ & 0.47 & 27 & 0.04 & Fort Union \\
\hline 12 & Ambassador oil & 1 Federal-J-Wyoming & WY & $16 \mathrm{~N}$ & $93 \mathrm{~W}$ & 14 & cuttings & coal & $3240-3260$ & 0.47 & 31 & 0.04 & Fort Union \\
\hline 12 & Ambassador oil & 1 Federal-J-Wyoming & WY & $16 \mathrm{~N}$ & $93 \mathrm{~W}$ & 14 & cuttings & coal & $4010-4020$ & 0.57 & 21 & 0.04 & Fort Union \\
\hline 12 & Ambassador oil & 1 Federal-J-Wyoming & WY & $16 \mathrm{~N}$ & $93 \mathrm{~W}$ & 14 & cuttings & coal & $5520-5600$ & 0.64 & 31 & 0.04 & Lance \\
\hline 12 & Ambassador oil & 1 Federal-J-Wyoming & WY & $16 \mathrm{~N}$ & $93 \mathrm{~W}$ & 14 & cuttings & coal & $6010-6060$ & 0.71 & 31 & 0.04 & Lance \\
\hline 12 & Ambassador oil & 1 Federal-J-Wyoming & WY & $16 \mathrm{~N}$ & $93 \mathrm{~W}$ & 14 & cuttings & coal & $9030-9040$ & 0.91 & 31 & 0.03 & Mesaverde \\
\hline 13 & Amoco Prod. Co. & 1 Bitter Creek II & WY & $16 \mathrm{~N}$ & $99 \mathrm{~W}$ & 22 & cuttings & coal & $3070-3080$ & 0.53 & 21 & 0.04 & Wasatch \\
\hline 13 & Amoco Prod. Co. & 1 Bitter Creek II & WY & $16 \mathrm{~N}$ & $99 \mathrm{~W}$ & 22 & cuttings & coal & $4000-4020$ & 0.53 & 21 & 0.05 & Wasatch \\
\hline 13 & Amoco Prod. Co. & 1 Bitter Creek II & WY & $16 \mathrm{~N}$ & $99 \mathrm{~W}$ & 22 & cuttings & coal & $5000-5020$ & 0.46 & 25 & 0.04 & Wasatch \\
\hline 13 & Amoco Prod. Co. & 1 Bitter Creek II & WY & $16 \mathrm{~N}$ & $99 \mathrm{~W}$ & 22 & cuttings & coal & $6000-6020$ & 0.63 & 27 & 0.04 & Fort Union \\
\hline 13 & Amoco Prod. Co. & 1 Bitter Creek II & WY & $16 \mathrm{~N}$ & $99 \mathrm{~W}$ & 22 & cuttings & coal & $7100-7110$ & 0.63 & 35 & 0.05 & Fort Union \\
\hline 13 & Amoco Prod. Co. & 1 Bitter Creek II & WY & $16 \mathrm{~N}$ & $99 \mathrm{~W}$ & 22 & cuttings & coal & $8000-8010$ & 0.78 & 27 & 0.05 & Fort Union \\
\hline 13 & Amoco Prod. Co. & 1 Bitter Creek II & WY & $16 \mathrm{~N}$ & $99 \mathrm{~W}$ & 22 & cuttings & coal & $8990-9000$ & 0.8 & 27 & 0.05 & Lance \\
\hline 13 & Amoco Prod. Co. & 1 Bitter Creek II & WY & $16 \mathrm{~N}$ & $99 \mathrm{~W}$ & 22 & cuttings & coal & $10000-10050$ & 0.71 & 29 & 0.09 & Lewis \\
\hline 13 & Amoco Prod. Co. & 1 Bitter Creek II & WY & $16 \mathrm{~N}$ & $99 \mathrm{~W}$ & 22 & cuttings & coal & $10980-11030$ & 1.13 & 31 & 0.05 & Mesaverde \\
\hline 13 & Amoco Prod. Co. & 1 Bitter Creek II & WY & $16 \mathrm{~N}$ & $99 \mathrm{~W}$ & 22 & cuttings & coal & $11820-11880$ & 1.2 & 29 & 0.07 & Mesaverde \\
\hline 13 & Amoco Prod. Co. & 1 Bitter Creek II & WY & $16 \mathrm{~N}$ & $99 \mathrm{~W}$ & 22 & cuttings & coal & $12800-12830$ & 1.42 & 31 & 0.07 & Mesaverde \\
\hline 14 & Texaco & 15 Table Rock Unit & WY & $18 \mathrm{~N}$ & $98 \mathrm{~W}$ & 2 & cuttings & coal & $1530-1560$ & 0.45 & 25 & 0.05 & Fort Union \\
\hline 14 & Texaco & 15 Table Rock Unit & WY & $18 \mathrm{~N}$ & $98 \mathrm{~W}$ & 2 & cuttings & coal & $2070-2130$ & 0.45 & 25 & 0.04 & Fort Union \\
\hline 14 & Texaco & 15 Table Rock Unit & WY & $18 \mathrm{~N}$ & $98 \mathrm{~W}$ & 2 & cuttings & coal & $2800-2830$ & 0.46 & 25 & 0.04 & Fort Union \\
\hline 14 & Texaco & 15 Table Rock Unit & WY & $18 \mathrm{~N}$ & $98 \mathrm{~W}$ & 2 & cuttings & coal & $3280-3310$ & 0.54 & 25 & 0.02 & Lance \\
\hline 14 & Texaco & 15 Table Rock Unit & WY & $18 \mathrm{~N}$ & $98 \mathrm{~W}$ & 2 & cuttings & coal & $4660-4750$ & 0.65 & 25 & 0.04 & Lance \\
\hline 14 & Texaco & 15 Table Rock Unit & WY & $18 \mathrm{~N}$ & $98 \mathrm{~W}$ & 2 & cuttings & coal & $6450-6480$ & 0.73 & 26 & 0.04 & Mesaverde \\
\hline 14 & Texaco & 15 Table Rock Unit & WY & $18 \mathrm{~N}$ & $98 \mathrm{~W}$ & 2 & cuttings & coal & $7050-7140$ & 0.72 & 25 & 0.04 & Mesaverde \\
\hline 14 & Texaco & 15 Table Rock Unit & WY & $18 \mathrm{~N}$ & $98 \mathrm{~W}$ & 2 & cuttings & coal & $7710-7770$ & 0.7 & 25 & 0.04 & Mesaverde \\
\hline 14 & Texaco & 15 Table Rock Unit & WY & $18 \mathrm{~N}$ & $98 \mathrm{~W}$ & 2 & cuttings & coal & $8110-8170$ & 0.68 & 25 & 0.04 & Mesaverde \\
\hline 15 & Amoco & 2 Tierney & WY & $19 \mathrm{~N}$ & $94 \mathrm{~W}$ & 15 & cuttings & coal & $1440-1470$ & 0.57 & 21 & 0.05 & Wasatch(?) \\
\hline 15 & Amoco & 2 Tierney & WY & $19 \mathrm{~N}$ & $94 \mathrm{~W}$ & 15 & cuttings & coal & $2440-2470$ & 0.45 & 25 & 0.05 & Fort Union \\
\hline 15 & Amoco & 2 Tierney & WY & $19 \mathrm{~N}$ & $94 \mathrm{~W}$ & 15 & cuttings & coal & $3150-3270$ & 0.61 & 21 & 0.04 & Fort Union \\
\hline 15 & Amoco & 2 Tierney & WY & $19 \mathrm{~N}$ & $94 \mathrm{~W}$ & 15 & cuttings & coal & $3690-3720$ & 0.61 & 21 & 0.03 & Fort Union \\
\hline 15 & Amoco & 2 Tierney & WY & $19 \mathrm{~N}$ & $94 \mathrm{~W}$ & 15 & cuttings & coal & $3980-4010$ & 0.58 & 25 & 0.04 & Fort Union \\
\hline 15 & Amoco & 2 Tierney & WY & $19 \mathrm{~N}$ & $94 \mathrm{~W}$ & 15 & cuttings & coal & $6950-7090$ & 0.69 & 27 & 0.05 & Lance \\
\hline 15 & Amoco & 2 Tierney & WY & $19 \mathrm{~N}$ & $94 \mathrm{~W}$ & 15 & cuttings & coal & $9660-9670$ & 0.92 & 31 & 0.04 & Mesaverde \\
\hline 15 & Amoco & 2 Tierney & WY & $19 \mathrm{~N}$ & $94 \mathrm{~W}$ & 15 & cuttings & coal & $10580-10600$ & 0.94 & 27 & 0.04 & Mesaverde \\
\hline 15 & Amoco & 2 Tierney & WY & $19 \mathrm{~N}$ & $94 \mathrm{~W}$ & 15 & cuttings & coal & $10880-10900$ & 0.91 & 31 & 0.03 & Mesaverde \\
\hline
\end{tabular}




\begin{tabular}{|c|c|c|c|c|c|c|c|c|c|c|c|c|c|}
\hline 15 & Amoco & 2 Tierney & WY & $19 \mathrm{~N}$ & $94 \mathrm{~W}$ & 15 & cuttings & coal & $11200-11210$ & 0.87 & 27 & 0.05 & Mesaverde \\
\hline 16 & Davis Oil Co. & 1 Dagger Unit & WY & $19 \mathrm{~N}$ & $107 \mathrm{~W}$ & 2 & cuttings & coal & $6500-6537$ & 0.58 & 27 & 0.02 & Fort Union \\
\hline 16 & Davis Oil Co. & 1 Dagger Unit & WY & $19 \mathrm{~N}$ & $107 \mathrm{~W}$ & 2 & cuttings & coal & $6982-7136$ & 0.68 & 29 & 0.05 & Fort Union \\
\hline 16 & Davis Oil Co. & 1 Dagger Unit & WY & $19 \mathrm{~N}$ & $107 \mathrm{~W}$ & 2 & cuttings & coal & $7567-7597$ & 0.7 & 27 & 0.03 & Fort Union \\
\hline 16 & Davis Oil Co. & 1 Dagger Unit & WY & $19 \mathrm{~N}$ & $107 \mathrm{~W}$ & 2 & cuttings & coal & $8096-8189$ & 0.67 & 29 & 0.07 & Lance \\
\hline 16 & Davis Oil Co. & 1 Dagger Unit & WY & $19 \mathrm{~N}$ & $107 \mathrm{~W}$ & 2 & cuttings & coal & $8649-8710$ & 0.71 & 31 & 0.06 & Mesaverde \\
\hline 16 & Davis Oil Co. & 1 Dagger Unit & WY & $19 \mathrm{~N}$ & $107 \mathrm{~W}$ & 2 & cuttings & coal & $9951-9983$ & 0.79 & 31 & 0.06 & Mesaverde \\
\hline 16 & Davis Oil Co. & 1 Dagger Unit & WY & $19 \mathrm{~N}$ & $107 \mathrm{~W}$ & 2 & cuttings & coal & 10163-10193 & 0.84 & 33 & 0.05 & Mesaverde \\
\hline 16 & Davis Oil Co. & 1 Dagger Unit & WY & $19 \mathrm{~N}$ & $107 \mathrm{~W}$ & 2 & cuttings & coal & $10780-107811$ & 0.97 & 33 & 0.05 & Mesaverde \\
\hline 16 & Davis Oil Co. & 1 Dagger Unit & WY & $19 \mathrm{~N}$ & $107 \mathrm{~W}$ & 2 & cuttings & coal & $16269-16331$ & 1.97 & 21 & 0.17 & Frontier \\
\hline & & & & & & & & & & & & & \\
\hline 17 & Mountain Fuel & 2 Bruff Unit & WY & $19 \mathrm{~N}$ & $112 \mathrm{~W}$ & 16 & cuttings & coal & $6390-6440$ & 0.62 & 29 & 0.05 & Fort Union \\
\hline 17 & Mountain Fuel & 2 Bruff Unit & WY & $19 \mathrm{~N}$ & $112 \mathrm{~W}$ & 16 & cuttings & coal & $7030-7090$ & 0.58 & 37 & 0.03 & Mesaverde \\
\hline 17 & Mountain Fuel & 2 Bruff Unit & WY & $19 \mathrm{~N}$ & $112 \mathrm{~W}$ & 16 & cuttings & coal & $11200-11230$ & 1.04 & 16 & 0.11 & Frontier \\
\hline 18 & Pacific Transmission & 3-12 Federal & WY & $22 \mathrm{~N}$ & $92 \mathrm{~W}$ & 12 & cuttings & coal & $1670-1730$ & 0.46 & 25 & 0.03 & Fort Union \\
\hline 18 & Pacific Transmission & 3-12 Federal & WY & $22 \mathrm{~N}$ & $92 \mathrm{~W}$ & 12 & cuttings & coal & $2510-2540$ & 0.49 & 31 & 0.04 & Fort Union \\
\hline 18 & Pacific Transmission & 3-12 Federal & WY & $22 \mathrm{~N}$ & $92 \mathrm{~W}$ & 12 & cuttings & coal & $3450-3480$ & 0.49 & 27 & 0.04 & Fort Union \\
\hline 18 & Pacific Transmission & 3-12 Federal & WY & $22 \mathrm{~N}$ & $92 \mathrm{~W}$ & 12 & cuttings & coal & $4030-4090$ & 0.57 & 25 & 0.05 & Fort Union \\
\hline 18 & Pacific Transmission & 3-12 Federal & WY & $22 \mathrm{~N}$ & $92 \mathrm{~W}$ & 12 & cuttings & coal & $4390-4450$ & 0.5 & 31 & 0.06 & Fort Union \\
\hline 18 & Pacific Transmission & 3-12 Federal & WY & $22 \mathrm{~N}$ & $92 \mathrm{~W}$ & 12 & cuttings & coal & $5230-5290$ & 0.52 & 31 & 0.03 & Fort Union \\
\hline 18 & Pacific Transmission & 3-12 Federal & WY & $22 \mathrm{~N}$ & $92 \mathrm{~W}$ & 12 & cuttings & coal & $6130-6190$ & 0.64 & 25 & 0.02 & Lance \\
\hline 18 & Pacific Transmission & 3-12 Federal & WY & $22 \mathrm{~N}$ & $92 \mathrm{~W}$ & 12 & cuttings & coal & $8080-8140$ & 0.62 & 31 & 0.04 & Lance \\
\hline 18 & Pacific Transmission & 3-12 Federal & WY & $22 \mathrm{~N}$ & $92 \mathrm{~W}$ & 12 & cuttings & coal & $9060-9080$ & 0.77 & 25 & 0.05 & Lance \\
\hline 18 & Pacific Transmission & 3-12 Federal & WY & $22 \mathrm{~N}$ & $92 \mathrm{~W}$ & 12 & cuttings & coal & $9790-9800$ & 0.83 & 35 & 0.03 & Lance \\
\hline 18 & Pacific Transmission & 3-12 Federal & WY & $22 \mathrm{~N}$ & $92 \mathrm{~W}$ & 12 & cuttings & coal & $10260-10270$ & 0.83 & 31 & 0.04 & Lance \\
\hline 18 & Pacific Transmission & 3-12 Federal & WY & $22 \mathrm{~N}$ & $92 \mathrm{~W}$ & 12 & cuttings & coal & $13130-13140$ & 1.35 & 35 & 0.05 & Mesaverde \\
\hline 19 & Ohio Oil Co. & Mud Lake 1 & WY & $23 \mathrm{~N}$ & $98 \mathrm{~W}$ & 12 & cuttings & coal & $1420-1460$ & 0.47 & 23 & 0.07 & Fort Union \\
\hline 19 & Ohio Oil Co. & Mud Lake 1 & WY & $23 \mathrm{~N}$ & $98 \mathrm{~W}$ & 12 & cuttings & coal & $1800-1860$ & 0.44 & 7 & 0.06 & Fort Union \\
\hline 19 & Ohio Oil Co. & Mud Lake 1 & WY & $23 \mathrm{~N}$ & $98 \mathrm{~W}$ & 12 & cuttings & coal & $2240-2260$ & 0.61 & 25 & 0.08 & Fort Union \\
\hline 19 & Ohio Oil Co. & Mud Lake 1 & WY & $23 \mathrm{~N}$ & $98 \mathrm{~W}$ & 12 & cuttings & coal & $3320-3345$ & 0.55 & 29 & 0.05 & Fort Union \\
\hline 19 & Ohio Oil Co. & Mud Lake 1 & WY & $23 \mathrm{~N}$ & $98 \mathrm{~W}$ & 12 & cuttings & coal & $3880-3900$ & 0.55 & 18 & 0.04 & Fort Union \\
\hline 19 & Ohio Oil Co. & Mud Lake 1 & WY & $23 \mathrm{~N}$ & $98 \mathrm{~W}$ & 12 & cuttings & coal & $4440-4460$ & 0.63 & 25 & 0.05 & Fort Union \\
\hline 19 & Ohio Oil Co. & Mud Lake 1 & WY & $23 \mathrm{~N}$ & $98 \mathrm{~W}$ & 12 & cuttings & coal & $5040-5060$ & 0.67 & 15 & 0.05 & Lance \\
\hline 19 & Ohio Oil Co. & Mud Lake 1 & WY & $23 \mathrm{~N}$ & $98 \mathrm{~W}$ & 12 & cuttings & coal & $6720-6750$ & 0.79 & 27 & 0.06 & Lance \\
\hline 19 & Ohio Oil Co. & Mud Lake 1 & WY & $23 \mathrm{~N}$ & $98 \mathrm{~W}$ & 12 & cuttings & coal & $7460-7510$ & 0.77 & 21 & 0.07 & Lance \\
\hline 19 & Ohio Oil Co. & Mud Lake 1 & WY & $23 \mathrm{~N}$ & $98 \mathrm{~W}$ & 12 & cuttings & coal & $9099-9100$ & 0.94 & 41 & 0.05 & Mesaverde \\
\hline 19 & Ohio Oil Co. & Mud Lake 1 & WY & $23 \mathrm{~N}$ & $98 \mathrm{~W}$ & 12 & cuttings & coal & $9140-9150$ & 0.95 & 25 & 0.08 & Mesaverde \\
\hline 19 & Ohio Oil Co. & Mud Lake 1 & WY & $23 \mathrm{~N}$ & $98 \mathrm{~W}$ & 12 & cuttings & coal & $9400-9420$ & 0.66 & 14 & 0.06 & Mesaverde \\
\hline
\end{tabular}




\begin{tabular}{|c|c|c|c|c|c|c|c|c|c|c|c|c|c|}
\hline 20 & Elf Aquataine & 33-32 McBride & WY & $24 \mathrm{~N}$ & $103 \mathrm{~W}$ & 32 & core & coal & 9067 & 0.9 & 41 & 0.03 & Frontier \\
\hline 21 & El Paso Nat. Gas & 1 Unit & WY & $24 \mathrm{~N}$ & $113 \mathrm{~W}$ & 11 & cuttings & coal & $3340-3400$ & 0.67 & 13 & 0.07 & Fort Union \\
\hline 21 & El Paso Nat. Gas & 1 Unit & WY & $24 \mathrm{~N}$ & $113 \mathrm{~W}$ & 11 & cuttings & coal & $4050-4060$ & 0.49 & 29 & 0.04 & Fort Union \\
\hline 21 & El Paso Nat. Gas & 1 Unit & WY & $24 \mathrm{~N}$ & $113 \mathrm{~W}$ & 11 & cuttings & coal & $4630-4730$ & 0.57 & 30 & 0.06 & Fort Union \\
\hline 21 & El Paso Nat. Gas & 1 Unit & WY & $24 \mathrm{~N}$ & $113 \mathrm{~W}$ & 11 & cuttings & coal & $5290-5390$ & 0.66 & 27 & 0.07 & Mesaverde \\
\hline 21 & El Paso Nat. Gas & 1 Unit & WY & $24 \mathrm{~N}$ & $113 \mathrm{~W}$ & 11 & cuttings & coal & $6050-6070$ & 0.58 & 31 & 0.05 & Mesaverde \\
\hline 22 & Southland Royalty & 1 Eaqles Nest Fed & WY & $25 \mathrm{~N}$ & $91 \mathrm{~W}$ & 29 & cuttings & coal & $6240-6330$ & 0.55 & 25 & 0.06 & Fort Union \\
\hline 22 & Southland Royalty & 1 Eagles Nest Fed. & WY & $25 \mathrm{~N}$ & $91 \mathrm{~W}$ & 29 & cuttings & coal & $7260-7380$ & 0.66 & 25 & 0.05 & Fort Union \\
\hline 22 & Southland Royalty & 1 Eagles Nest Fed. & WY & $25 \mathrm{~N}$ & $91 \mathrm{~W}$ & 29 & cuttings & coal & $8640-8700$ & 0.68 & 25 & 0.03 & Fort Union \\
\hline 22 & Southland Royalty & 1 Eagles Nest Fed. & WY & $25 \mathrm{~N}$ & $91 \mathrm{~W}$ & 29 & cuttings & coal & $9060-9070$ & 0.68 & 25 & 0.03 & Fort Union \\
\hline 22 & Southland Royalty & 1 Eagles Nest Fed. & WY & $25 \mathrm{~N}$ & $91 \mathrm{~W}$ & 29 & cuttings & coal & $9270-9300$ & 0.74 & 25 & 0.04 & Fort Union \\
\hline 22 & Southland Royalty & 1 Eagles Nest Fed. & WY & $25 \mathrm{~N}$ & $91 \mathrm{~W}$ & 29 & cuttings & coal & $11750-11840$ & 0.81 & 25 & 0.05 & Lance \\
\hline 22 & Southland Royalty & 1 Eagles Nest Fed. & WY & $25 \mathrm{~N}$ & $91 \mathrm{~W}$ & 29 & cuttings & coal & $13180-13330$ & 0.98 & 25 & 0.05 & Lance \\
\hline 22 & Southland Royalty & 1 Eagles Nest Fed. & WY & $25 \mathrm{~N}$ & $91 \mathrm{~W}$ & 29 & cuttings & coal & $13840-13900$ & 1.21 & 25 & 0.05 & Lance \\
\hline 22 & Southland Royalty & 1 Eagles Nest Fed. & WY & $25 \mathrm{~N}$ & $91 \mathrm{~W}$ & 29 & cuttings & coal & $14730-14760$ & 1.4 & 27 & 0.05 & Lance \\
\hline 22 & Southland Royalty & 1 Eagles Nest Fed. & WY & $25 \mathrm{~N}$ & $91 \mathrm{~W}$ & 29 & cuttings & coal & $15390-15410$ & 1.46 & 27 & 0.04 & Lance \\
\hline 23 & American Hunter & Enterprise 1-A & WY & $25 \mathrm{~N}$ & $107 \mathrm{~W}$ & 30 & cuttings & coal & $4420-4460$ & 0.53 & 25 & 0.02 & Fort Union \\
\hline 23 & American Hunter & Enterprise 1-A & WY & $25 \mathrm{~N}$ & $107 \mathrm{~W}$ & 30 & cuttings & coal & $5250-5350$ & 0.58 & 25 & 0.03 & Fort Union \\
\hline 23 & American Hunter & Enterprise 1-A & WY & $25 \mathrm{~N}$ & $107 \mathrm{~W}$ & 30 & cuttings & coal & $6310-6320$ & 0.64 & 25 & 0.03 & Fort Union \\
\hline 23 & American Hunter & Enterprise 1-A & WY & $25 \mathrm{~N}$ & $107 \mathrm{~W}$ & 30 & cuttings & coal & $7090-7170$ & 0.7 & 25 & 0.04 & Fort Union \\
\hline 23 & American Hunter & Enterprise 1-A & WY & $25 \mathrm{~N}$ & $107 \mathrm{~W}$ & 30 & cuttings & coal & $8310-8330$ & 0.77 & 25 & 0.04 & Mesaverde \\
\hline 23 & American Hunter & Enterprise 1-A & WY & $25 \mathrm{~N}$ & $107 \mathrm{~W}$ & 30 & cuttings & coal & $8530-8540$ & 0.83 & 25 & 0.04 & Mesaverde \\
\hline 23 & American Hunter & Enterprise 1-A & WY & $25 \mathrm{~N}$ & $107 \mathrm{~W}$ & 30 & cuttings & coal & $10270-10280$ & 1.04 & 25 & 0.05 & Mesaverde \\
\hline 23 & American Hunter & Enterprise 1-A & WY & $25 \mathrm{~N}$ & $107 \mathrm{~W}$ & 30 & cuttings & coal & $10610-10620$ & 1.13 & 25 & 0.03 & Mesaverde \\
\hline 24 & Nat. Gas Corp. of Calif. & Fontenelle 22-22B & WY & $26 \mathrm{~N}$ & $112 \mathrm{~W}$ & 22 & core & coal & 8816 & 1.02 & 35 & 0.04 & Frontier \\
\hline 25 & Davis Oil Co. & Sugar Loaf Unit 1 & WY & $27 \mathrm{~N}$ & $109 W$ & 7 & cuttings & coal & $7050-7200$ & 0.65 & 25 & 0.05 & Lance \\
\hline 25 & Davis Oil Co. & Sugar Loaf Unit 1 & WY & $27 \mathrm{~N}$ & $109 \mathrm{~W}$ & 7 & cuttings & coal & $7440-7840$ & 0.62 & 25 & 0.06 & Lance \\
\hline 25 & Davis Oil Co. & Sugar Loaf Unit 1 & WY & $27 \mathrm{~N}$ & $109 \mathrm{~W}$ & 7 & cuttings & coal & $8640-8830$ & 0.77 & 25 & 0.05 & Mesaverde \\
\hline 25 & Davis Oil Co. & Sugar Loaf Unit 1 & WY & $27 \mathrm{~N}$ & $109 \mathrm{~W}$ & 7 & cuttings & coal & $9240-9450$ & 0.71 & 25 & 0.04 & Mesaverde \\
\hline 25 & Davis Oil Co. & Sugar Loaf Unit 1 & WY & $27 \mathrm{~N}$ & $109 \mathrm{~W}$ & 7 & cuttings & coal & $9690-9830$ & 0.76 & 25 & 0.04 & Mesaverde \\
\hline 25 & Davis Oil Co. & Sugar Loaf Unit 1 & WY & $27 \mathrm{~N}$ & $109 W$ & 7 & cuttings & coal & $9450-9650$ & 0.71 & 25 & 0.07 & Mesaverde \\
\hline 25 & Davis Oil Co. & Sugar Loaf Unit 1 & WY & $27 \mathrm{~N}$ & $109 W$ & 7 & cuttings & coal & $10060-10110$ & 0.81 & 25 & 0.05 & Mesaverde \\
\hline 25 & Davis Oil Co. & Sugar Loaf Unit 1 & WY & $27 \mathrm{~N}$ & $109 \mathrm{~W}$ & 7 & cuttings & coal & $11380-11420$ & 0.75 & 20 & 0.08 & Mesaverde \\
\hline 25 & Davis Oil Co. & Sugar Loaf Unit 1 & WY & $27 \mathrm{~N}$ & $109 W$ & 7 & cuttings & coal & $15520-15650$ & 1.98 & 25 & 0.08 & Frontier \\
\hline 26 & Mountain Fuel & 1 Three Bridges & WY & $30 \mathrm{~N}$ & $110 \mathrm{~W}$ & 7 & cuttings & coal & $2220-2280$ & 0.52 & 31 & 0.03 & Wasatch(?) \\
\hline 26 & Mountain Fuel & 1 Three Bridges & WY & $30 \mathrm{~N}$ & $110 \mathrm{~W}$ & 7 & cuttings & coal & $3310-3330$ & 0.53 & 31 & 0.04 & Wasatch(?) \\
\hline
\end{tabular}




\begin{tabular}{|c|c|c|c|c|c|c|c|c|c|c|c|c|c|}
\hline 26 & Mountain Fuel & 1 Three Bridges & WY & $30 \mathrm{~N}$ & $110 \mathrm{~W}$ & 7 & cuttings & coal & $6780-6830$ & 0.63 & 27 & 0.06 & Fort Union \\
\hline 26 & Mountain Fuel & 1 Three Bridges & WY & $30 N$ & $110 \mathrm{~W}$ & 7 & cuttings & coal & $8600-8700$ & 0.72 & 27 & 0.06 & Lance \\
\hline 26 & Mountain Fuel & 1 Three Bridges & WY & $30 \mathrm{~N}$ & $110 \mathrm{~W}$ & 7 & cuttings & coal & $9440-9490$ & 0.85 & 27 & 0.1 & Lance \\
\hline
\end{tabular}

